\title{
CUIDADO A LOS ADOLESCENTES INSTITUCIONALIZADOS: PERSPECTIVA DE LAS MADRES CUIDADORAS
}

\author{
CARING FOR INSTITUTIONALIZED ADOLESCENTS: THE \\ CAREGIVER'S PERSPECTIVE
}

\section{CUIDADO AO ADOLESCENTES INSTITUCIONALIZADOS: PERSPECTIVA DOS CUIDADORES}

\author{
Priscilla Marysol Gamarra Quiroz* \\ Rosa Jeuna Díaz Manchay** \\ Lisseth Dolores Rodríguez CRuz*** \\ Sonia Tejada Muñoz ${ }^{* * * *}$ \\ Mirtha Flor Cervera Vallejos***** \\ Antonieta De Jesús Banda Pérez ${ }^{* * * * * *}$ \\ Sonia Celedonia Huyhua Gutiérrez ${ }^{* * * * * * *}$
}

\begin{abstract}
RESUMEN
Objetivo: Describir el cuidado a los adolescentes institucionalizados desde la perspectiva de madres cuidadoras en un centro de atención residencial de Chiclayo, Perú. Material y Método: Investigación cualitativa descriptiva, con muestreo no probabilístico, por conveniencia y saturación, cuyas participantes, previo consentimiento informado, fueron 10 madres cuidadoras de un centro de atención residencial y a quienes se les aplicó una entrevista semiestructurada, validada mediante juicio de tres expertos. El rigor se sustentó en los criterios de credibilidad, auditabilidad y transferibilidad. Resultados: Se identificaron 3 categorías: a) Cumplimiento de los requerimientos nutricionales y monitorización de la higiene personal, b) Afecto y comunicación asertiva para reeducar en valores y c) Apoyo en las tareas del rol de la vida diaria. Conclusión: Las madres cuidadoras
\end{abstract}

*Licenciada en Enfermería. Centro de Atención Residencial San Juan Bosco - INABIF. Chiclayo, Perú. ORCID: https://orcid. org/0000-0002-2828-1698 Email: priskax87@gmail.com

** Doctora en Ciencias de Enfermería. Escuela de Enfermería de la Universidad Católica Santo Toribio de Mogrovejo, Chiclayo, Perú. ORCID: https://orcid.org/0000-0002-2333-7963 Email: rdiaz@usat.edu.pe Autora de correspondencia

*** Maestra en Bioética. Escuela de Enfermería de la Universidad Católica Santo Toribio de Mogrovejo, Chiclayo, Perú. ORCID: https://orcid.org/0000-0003-1742-9498 Email: 1rodriguez@usat.edu.pe

**** Doctora en Ciencias de Enfermería. Escuela de Enfermería de la Universidad Nacional Toribio Rodríguez de Mendoza, Chachapoyas, Perú. ORCID: https://orcid.org/0000-0002-1181-8540 Email: sonia.tejada@untrm.edu.pe

***** Doctora en Ciencias de Enfermería. Docente de la Universidad Católica Santo Toribio de Mogrovejo, Chiclayo, Perú. ORCID: https://orcid.org/0000-0002-4972-1787 Email: mcervera@usat.edu.pe

******Estudiante de Doctorado en Ciencias de Enfermería, Campus Celaya Salvatierra Universidad de Guanajuato. Docente de la Facultad de Enfermería y Nutrición de la Universidad Autónoma de San Luis Potosí, México. ORCID: https://orcid.org/00000001-9586-0641 Email: antonieta.banda@uaslp.mx

*******Licenciada en Enfermería. Escuela de Enfermería de la Universidad Nacional Toribio Rodríguez de Mendoza, Chachapoyas, Perú. ORCID: https://orcid.org/0000-0003-4823-2778 Email: sonia.huyhua@untrm.edu.pe 
asumen la función como lo haría una madre biológica, coordinando con todos los profesionales que allí trabajan, y en equipo intentan formar o reformar a un adolescente separado de su familia consanguínea por orden judicial al carecer de un entorno favorable para su crecimiento y desarrollo. De este modo realizan un conjunto de actividades para satisfacer las necesidades primordiales y afectivas, inculcan disciplina y valores, y el cumplimiento del rol diario de vida para su autosuperación. También propician actividades recreativas y deportivas. Incluso hay indicios que fortalecen la fe a través de la oración y actúa para coordinar siempre con todos los profesionales que allí trabajan, en especial con la enfermera, quien es la responsable de velar por la salud integral de estos adolescentes.

Palabras clave: Cuidado en custodia; Adolescente; Albergue; Investigación cualitativa.

\begin{abstract}
Objective: To describe the care for institutionalized adolescents from the perspective of caregiving women in a residential care center in Chiclayo, Peru. Material and Method: Descriptive qualitative research, with nonprobabilistic sampling, using convenience and saturation. Participants consisted of 10 caregiving women of a residential care center, who signed prior informed consent and responded a semi-structured interview validated by three experts. Rigor was based on the criteria of credibility, auditability and transferability. Results: 3 categories were identified: a) Compliance with nutritional requirements and monitoring of personal hygiene, b) Affection and assertive communication to re-educate in values and c) Support in daily life tasks. Conclusion: Caregivers act as biological mothers, coordinating with other professionals working at the care center. They try to educate or re-educate an adolescent separated from his or her biological family after a court ruling decision due to lack of a favorable environment for his or her growth and development. In this way, they carry out different activities to satisfy the primary and affective needs, instill discipline and values, as well as encourage self-improvement through daily tasks. They also promote recreational and sports activities. There are even indications that caregivers strengthen faith through prayer and work in coordination with other professionals, especially with the nurses who are responsible for the general health of adolescents.
\end{abstract}

Key words: Custodial care; Adolescent; Shelter; Qualitative research.

\title{
RESUMO
}

Objetivo: Descrever o cuidado a adolescentes institucionalizados na perspectiva de cuidadores de um centro residencial de saúde em Chiclayo, Peru. Material e Método: Pesquisa qualitativa descritiva, com amostragem não probabilística, por conveniência e saturaçáo, cujos participantes, com consentimento informado prévio, foram 10 cuidadores de um centro de assistência residencial e aos quais foi aplicada uma entrevista semiestruturada, validada por julgamento de três especialistas. O rigor baseou-se nos critérios de credibilidade, auditabilidade e transferibilidade. Resultados: Foram identificadas 3 categorias: a) Cumprimento das necessidades nutricionais e acompanhamento da higiene pessoal, b) Afeto e comunicação assertiva para reeducar em valores e c) Apoio nas tarefas do papel da vida diária. Conclusão: As mães cuidadoras assumem o papel de mãe biológica, articulandose com todos os profissionais que ali atuam, e em equipe procuram formar ou reformar o adolescente separado de sua família consanguínea por ordem judicial por falta de um ambiente favorável para seu crescimento e desenvolvimento. Dessa forma, realizam um conjunto de atividades para a satisfação das necessidades primordiais e afetivas, inculcam disciplina e valores, e o cumprimento do papel cotidiano da vida para o seu crescimento pessoal. Eles também promovem atividades recreativas e esportivas. Há até sinais que fortalecem a fé por meio da oração e agem em articulação sempre com todos os profissionais que ali trabalham, principalmente com o enfermeiro, que é responsável por zelar pela saúde integral desses adolescentes.

Palavras-chave: Cuidados de custódia; Adolescente; Albergue; Pesquisa qualitativa.

Fecha de recepción: 22/01/2021

Fecha de aceptación: 28/05/2021 


\section{INTRODUCCIÓN}

La problemática de infantes y adolescentes de ambos sexos que carecen del cuidado directo de sus familias en el trayecto de su crianza muestra derechos vulnerados en sus propios grupos familiares, al ser sometidos a violencia intrafamiliar, maltrato, abandono y otras situaciones que pueden dañar su salud física y mental. Frente a ello, una alternativa es institucionalizarse y vivir en los centros de $\operatorname{acogida}^{(1,2)}$. Según datos del Plan Nacional Integral de Bienestar Familiar (INABIF) de Perú, aproximadamente 17.000 niños(as) y adolescentes abandonados son ubicados en los Centros de Atención Residencial (CAR), con el fin de alcanzar el bienestar y desarrollo personal, potenciar sus capacidades para que cuando cumplan la mayoría de edad sean personas productivas para la sociedad, la comunidad y la familia ${ }^{(3)}$.

En ese sentido, las instituciones de esta índole tienen que ser propicias para generar un ambiente cálido e inspirador, similar a un ambiente familiar positivo, a fin de: establecer lazos seguros y sanos, reconstruir los vínculos con su familia, se relacionen y conecten con otras redes sociales para que vean referencias adecuadas a su etapa de $\operatorname{vida}^{(1)}$. Sin embargo, algunas investigaciones reportan un déficit en los infantes y adolescentes que son institucionalizados frente a los que están en contextos familiares, en aspectos que abarcan el comportamiento, las competencias psicosociales y el crecimiento y desarrollo ${ }^{(2,4,5)}$. Esta situación llega a ser compleja cuando los residentes son adolescentes, pues atraviesan una de las etapas de la vida más complicada por los diversos cambios y conflictos que enfrentan ${ }^{(6)}$; realidad que los cuidadores y profesionales que laboran en estas instituciones deben reconocer y actuar.

$\mathrm{Al}$ respecto, Vanegas y Castrillón ${ }^{(7)}$ encontraron que, si bien los cuidadores en estas instituciones tratan de ser contenedores emocionales, no lo consiguen del todo, por la movilidad o cambio del personal, la falta de recursos humanos y logísticos, impidiendo una figura permanente o modelo estable para establecer un vínculo profundo y duradero. Es imperante que el adolescente viva en un ambiente armónico que le permita un desarrollo biológico, emocional y cognitivo, de modo que descubra y potencie sus habilidades, superando los traumas vividos.

En los CAR, los adolescentes son atendidos por un equipo multidisciplinario, en especial están a cargo de las "madres cuidadoras", quienes desempeñan la función como lo haría una madre biológica. Esta realidad comprende un reto y un nuevo espacio para Enfermería, donde trabaja con el mismo adolescente residente (AR), la familia biológica y la madre sustituta; ya que, si la madre sustituta no cumple con sus funciones, los adolescentes no desarrollarían sus capacidades para convivir dentro de un ambiente externo o reintegrados en la sociedad, o peor aún, cuando formen su propia familia.

Las madres cuidadoras son pieza fundamental en la vida de los adolescentes y en el comportamiento que estos van a desarrollar como ciudadanos. Teniendo en cuenta que por los cambios propios de la adolescencia, suelen aflorar miedos e inquietudes sobre sus orígenes o su situación actual, la actuación de las madres cuidadoras es un soporte positivo en el actuar de los adolescentes. De igual forma, al ser un ámbito con presencia de poblaciones vulnerables, como es el caso de los adolescentes, que es un grupo humano muchas veces desatendido y poco comprendido, la presencia y cuidados del profesional de enfermería es encomiable.

Por ello el objetivo de esta investigación fue describir el cuidado a los adolescentes institucionalizados desde la perspectiva de las madres cuidadoras en un centro de atención residencial de Chiclayo, Perú.

\section{MATERIAL Y MÉTODO}

Esta investigación tuvo un enfoque cualitativo descriptivo $^{(8)}$ y se realizó en el Centro de Atención Residencial San Juan Bosco (CAR-SJB) del INABIF en Chiclayo, Perú. Esta institución tiene un área de $20.000 \mathrm{~m}^{2}$ y una cuarta parte construida con la infraestructura de una casa; cuenta con 5 ambientes identificados por un valor (amistad, solidaridad, paz, caridad y amor); cuentan con sala, baños, área de estudio, habitaciones y sala de espera. Además, tienen un área administrativa, un almacén, una carpintería, una biblioteca, una 
sala de cómputo, una sala de reuniones para los cuidadores y un biohuerto que todos comparten. Residen 36 adolescentes varones de 13 a 18 años, sin cuidados parentales e ingresados por una orden del juzgado de familia.

El muestreo por conveniencia y saturación abarcó el total de las diez trabajadoras "madres cuidadoras" de la institución. Cabe señalar que ninguna madre se negó a participar en este estudio.

Se utilizó la entrevista semiestructurada ${ }^{(9)}$ elaborada por las investigadoras, a partir de los antecedentes y bases teóricas, validada mediante juicio de tres expertos, representados por enfermeras especialistas en el tema y en investigación cualitativa. Para evaluar la comprensión de las preguntas por las participantes del estudio, se realizó previamente una prueba piloto en un CAR que tuvo características similares.

Los datos se recolectaron entre julio y agosto de 2018, previa aprobación de la escuela de postgrado de la Universidad Católica Santo Toribio de Mogrovejo, Perú y la autorización del director del CAR-SJB para la aplicación de las entrevistas. Una vez identificadas las madres cuidadoras se les explicó los objetivos del estudio y se solicitó la firma del consentimiento informado. Con la finalidad de proteger su identidad fueron identificadas con códigos (M1 hasta M10). Según su disponibilidad, se acordó la fecha y la hora para realizar la entrevista. El tiempo de cada entrevista abarcó un promedio de $25 \mathrm{~min}$, las cuales fueron grabadas, luego transcritas y a continuación mostradas a cada una de las madres para confirmar que los datos eran correctos.

El procesamiento de los datos fue de manera manual, mediante el análisis de contenido ${ }^{(9)}$, que permitió la comprensión y análisis a profundidad de datos cualitativos, en sus tres momentos: 1) Preanálisis: se organizaron las entrevistas de los participantes en archivos de Word, luego se hizo lectura y relectura para comprender e identificar las ideas principales en cada pregunta y en cada entrevista. Además, se fue quitando las ideas que no correspondían al tema de estudio; 2) Codificación: Se ubicaron los párrafos o grupos de párrafos que expresan una idea o un concepto central para obtener las unidades de significados temáticos que fueron consideradas como quince categorías preliminares que fueron fichadas por el método de coloreo y subrayado; 3) Categorización: Se agruparon las unidades temáticas por similitud en cuanto a su significado obteniéndose tres categorías.

Entre los aspectos éticos ${ }^{(10)}$, las madres participantes en la investigación no sufrieron daño físico, emocional, eligieron de manera voluntaria participar, firmaron el consentimiento informado, y su identidad fue resguardada. Del mismo modo, se consideraron los siguientes criterios de rigor científico $^{(8)}$ : a) Credibilidad: después de transcritas las entrevistas en procesador de texto Word, fueron retornadas a cada una de las madres cuidadoras vía WhatsApp, de modo que dieron su conformidad con lo expresado; b) Auditabilidad: para ello se resguardó la información recolectada por dos años, luego de ese período se eliminó; c) Transferibilidad: al describir el escenario donde se desarrolló la investigación y las características de los sujetos de estudio, con la finalidad de que otros investigadores lo apliquen en su realidad.

Cabe destacar que todo el equipo de investigación de este estudio tiene experiencia en investigaciones cualitativas, cinco de ellas son docentes universitarias y asesoras de tesis y como mínimo han ejecutado una investigación en personal de salud. Además, una de las autoras es enfermera y trabaja en la institución, pero no formó parte del grupo de participantes de la investigación porque los datos se recolectaron en el mes de sus vacaciones.

\section{RESULTADOS}

Características de las madres cuidadoras: El grupo de participantes estuvo integrado por una docente de inicial, siete docentes de primaria, una socióloga y una psicóloga. Sus edades oscilaron entre 24 y 58 años, todas casadas, con hijos, laboraban 12 h diarias (de 7 a.m. a 7 p.m.) por 4 días a la semana. Ellas cumplen la función de una madre común, y por las noches los adolescentes están bajo la responsabilidad del tutor (cumple el rol de padre).

El cuidado a los adolescentes institucionalizados desde la perspectiva de las madres cuidadoras: $L o s$ adolescentes varones residentes del CAR-SJB, por 
su etapa de vida, costumbres o comportamientos, a veces inadecuados, traídos desde un hogar donde vivencian carencia de afecto y conflictos, requieren mucha atención para satisfacer sus necesidades físicas, afectivas y disciplinarias, entre ellas: formación en valores, orientación en el cumplimiento de tareas escolares y del rol cotidiano y enseñanza de oficios o tareas del hogar eficaces para la vida. Todas estas atenciones son encargadas a las madres cuidadoras del CAR, quienes, además, coordinan con todos los profesionales que allí trabajan, en especial con la nutricionista, enfermera, y el tutor (padre).

De la agrupación de las unidades temáticas se obtuvieron tres categorías, ilustradas a continuación con los discursos más significativos:

a) Cumplimiento de los requerimientos nutricionales y monitorización de la higiene personal: Las madres cuidadoras, además de las labores domésticas, son responsables de cumplir con las dietas indicadas por la nutricionista; la institución brinda los alimentos y ellas lo cocinan, hacen algunos gustos para que los adolescentes residentes consuman la ración que les corresponde. Además, promueven el lavado de manos y algunas madres motivan a bendecir los alimentos antes de ingerirlos:

Nosotras lavamos, planchamos, tenemos limpia la casa...cocinamos el menú que nos da la nutricionista, nos dan los alimentos y ya cada uno le da su sazón, o alguna dieta especial si asi lo indican... antes de comer se laven las manos y rezamos (M2, M6).

Le damos una dieta balanceada, tratamos que aprendan a comer saludable como las verduras $y$ frutas... les hago sus gustos como un postre, o si a alguien no le gusta la cebolla, la saco al momento que va a comer... lo importante es que coman (M4, M7).

También cuido su aspecto físico, que den una buena imagen...vemos que su cabello este recortado, que se laven los dientes, se bañen porque algunos no quieren bañarse (M1).

Superviso que se bañen bien con shampoo y jabón, se laven los dientes, se peinen, recorten sus uñas, usen ropa limpia...que tengan las manos limpias... sabemos lo importante que es la higiene tal como nos explica la enfermera (M3, M5).

Las madres cuidadoras evidencian responsabilidad directa de supervisar la higiene personal de los adolescentes, tales como: el baño, el cepillado de dientes, el recorte de pelo y uñas, los materiales de aseo personal y ropa limpia para que estén presentables, siguiendo las indicaciones dadas por la enfermera, todo en beneficio de mantener una salud óptima.

b) Afecto y comunicación asertiva para reeducar en valores: Las madres cuidadoras están pendientes del estado emocional de los adolescentes, si detectan alguna alteración en el comportamiento, lo informan a la psicóloga o a la enfermera, y reportan al tutor (padre) para su seguimiento. La comunicación con los adolescentes es vital, y lo hacen mientras comparten el desayuno, almuerzo o la cena, o cuando lo consideren necesario. Se rescata que a pesar de no ser sus madres de consanguinidad entablan una buena relación, brindan afecto y llegan a formar lazos estrechos de confianza:

Cuidar la estabilidad emocional del residente... me encargo de velar por ellos, de estar pendientes donde están, que están haciendo, cuando los veo deprimidos, me siento a conversar con ellos, me cuentan sus problemas... si vemos que no es suficiente, recurrimos a psicología, e informamos a la enfermera $y$ al tutor para seguimiento (M3).

Ellos son como nuestros hijos, compartimos anécdotas...necesitan afecto y ser escuchados... les hablo, les oriento, tratamos de reeducarlos o reorientarlos en valores, pues en su hogar tuvieron carencias de esto...les inculcamos el respeto, la responsabilidad, la puntualidad, la honestidad, valores que le servirá para su futuro (M6, M10).

La comunicación en fundamental y para ello nos ganamos su confianza... tratamos de educarlos en buenos modales y enseñándoles los valores, los mandamientos de Dios (M8).

Las madres cuidadoras les enseñan qué es lo bueno y lo malo, les demuestran cuáles son los valores con la práctica en el día a día; si bien es cierto educar es difícil, reeducar es más, porque necesitan desaprender conductas que no son favorables para adquirir valores como la responsabilidad, la puntualidad, la honestidad; también algunas madres enseñan los mandamientos de Dios, o un acercamiento a la vida espiritual.

c) Apoyo en las tareas del rol de vida diaria: Las madres cuidadoras de los residentes adolescentes 
juntamente con el tutor (función de padre) son responsables de hacer cumplir el rol de vida diario (actividades programadas por la institución desde que se levantan hasta que duermen). Destaca la habilidad que tienen para enseńarles a ser responsables, mantener limpia su habitación y casa, a realizar quehaceres del hogar con la finalidad de que sean independientes y logren subsistir si vivieran solos. De esta manera, aprenden a lavar, planchar y ordenar su ropa; limpiar los baños y el hogar en general; combinado con actividades de jardinería como regar las plantas, arreglar la tierra y sembrar:

Se le enseña a lavar y planchar su ropa, y a limpiar: baños, pasadizos, salas, jardín, cocina, lavandería y almacenes... realizan la limpieza de sus habitaciones y organizar la ropa en sus roperos, riegan las plantas, arreglen la tierra y siembran, dan mantenimiento a las áreas verdes, bajo nuestra supervisión o del tutor... intentamos que lo aprendan bien como un oficio para que cuando salgan de la institución puedan trabajar en ello (M5).

Vemos sus fortalezas, algunos tienen inclinación por la cocina, les sacamos su carnet de salubridad y se les tiene de apoyo en la cocina, y cuando reúnen las condiciones para poder estudiar fuera del centro, la asistenta social busca becas para gastronomia (M9).

Les ayudamos con el reforzamiento escolar en algún tema que no está dominado, o los apoyamos a desarrollar alguna tarea, usamos los libros o la computadora de la biblioteca de la institución... cuando culminan sus tareas del colegio pueden subir a la sala de star y ver televisión... juegan futbol, vóley y básquet bajo la supervisión del tutor (M10).

Además, evidencian la facilidad para identificar fortalezas o talentos relacionados con alguna actividad $\mathrm{u}$ oficio en particular, como el caso de la gastronomía o la jardinería y comunican a la asistenta social para buscarles becas de estudios. En este rol de vida diario, es encomiable su preocupación para que salgan bien en el colegio, y les ayudan reforzándoles algunas clases o los apoyan con algunas tareas y para ello usan libros y computadoras en la biblioteca del CAR. También es importante rescatar que les permiten distraerse y ver televisión; unido a las actividades deportivas, donde juegan fútbol, vóleibol y básquetbol, pues la institución tiene dos canchas deportivas y estas actividades las supervisa el tutor.

\section{DISCUSIÓN}

Las madres cuidadoras de los adolescentes residentes del CAR-SJB, además de las labores domésticas, son responsables de preparar las dietas indicadas por la nutricionista, promueven hábitos saludables de alimentación (consumo de verduras, frutas y agua), y el lavado de manos. También, indica la enfermera, inculcan buenos hábitos de higiene como el baño diario, el cepillado dental después de las comidas, el recorte de uñas y del pelo, y el uso de ropa limpia, para que tengan buena imagen personal. Resultados corroborados con el estudio de Sánchez, Castro, Cantor y Bolaños ${ }^{(2)}$, al concluir que los cuidadores de niños institucionales se ocupan principalmente de cubrir necesidades básicas como la alimentación y la higiene.

Las madres cuidadoras están pendientes del estado emocional, informan si observan alguna alteración en el comportamiento a la psicóloga, la enfermera, y al tutor (padre), para tratamiento y seguimiento; promueven una buena relación, brindan afecto y llegan a formar lazos estrechos de confianza, pues la mayoría proviene de hogares disfuncionales y tienen carencias afectivas o conductas agresivas. Los niños y adolescentes institucionalizados son vulnerables, y necesitan superar el sentimiento de abandono $^{(2)}$, y esto es logrado cuando los cuidadores brindan apoyo emocional. Este apoyo es aceptado por los adolescentes porque desean ser escuchados y comprendidos, buscan la manera de resolver sus conflictos, intentan saber cómo equilibrar la vida con respecto a lo que sienten y lo que quieren $\operatorname{ser}^{(11)}$.

El cuidado de las madres cuidadoras afecta directamente a los niños/adolescentes institucionalizados. Mota y Matos $^{(12)}$ asienten que la percepción de los cuidadores de un vínculo emocional cercano está asociada con la empatía percibida y la capacidad de respuesta hacia los adolescentes. Así, la calidad del apego de los cuidadores, como facilitador de habilidades emocionales e interpersonales, ayuda a crear una relación de calidad con los adolescentes institucionalizados. Aun así, los hallazgos de Pinchover y Attar-Schwartz ${ }^{(13)}$ revelan una asocia- 
ción negativa significativa entre el clima social percibido por los adolescentes institucionalizados y las dificultades generales de adaptación. Por ello, se recomienda la capacitación programada de los cuidadores en salud mental, porque favorecerá el incremento del apoyo individual, así como el fomento y mejoras de los vínculos entre cuidador, niños y adolescentes institucionalizados ${ }^{(14,15)}$.

Las madres cuidadoras en este estudio inculcan valores, pero algunos adolescentes tienen conductas aprehendidas en casa, y son irrespetuosos, aspectos propios también de su desarrollo, ya que tienden a contradecir todo. Algunos investigadores ${ }^{(16,17)}$ señalan que en víctimas de maltrato existe una alta prevalencia de problemas emocionales y de comportamiento, congruentes con las experiencias tempranas adversas y los factores de riesgo, expuestos de forma peligrosa. Por ello, resulta ineludible llegar a establecer mecanismos para asegurar la detección de problemas de salud mental en este colectivo. El desarrollo de instrumentos adaptados y sensibles a sus particularidades y la educación de los profesionales sobre los tipos de trastornos que presentan estos jóvenes, como también la necesidad de mantener una vigilancia constante de sus cuidadores, ayudará a estar alerta para reportar a tiempo cualquier problema de salud mental.

Si bien es cierto educar es difícil, reeducar es más porque de lo que se trata es de que desaprendan conductas que no son favorables y practiquen valores como la responsabilidad, la puntualidad y la honestidad. Como en un hogar normal, las madres cuidadoras mantienen su preocupación por el rendimiento académico de los adolescentes institucionalizados y les hacen seguimiento para verificar el cumplimiento de sus tareas. En la revisión de Steels y Simpson ${ }^{(14)}$ se destaca que el cuidado residencial tiene un efecto tanto positivo como negativo en las emociones de los niños, en su desarrollo social y conductual, su salud mental y su progreso académico. De esta manera, los métodos de crianza proporcionados por los cuidadores ofrecerán un impacto positivo o negativo en el niño o adolescente, lo cual afectará directa o indirectamente el rendimiento académico ${ }^{(18)}$. También, las experiencias tempranas de estrés promoverán el desarrollo neurológico caracterizado por déficits emocionales y cognitivos ${ }^{(19)}$.
Estas circunstancias propician un análisis reflexivo sobre los hogares de acogida como medida de protección y restitución de los derechos de los niños, niñas y adolescentes, a fin de realizar las correcciones necesarias para que crezcan en un ambiente sano, que les permita desarrollarse de manera armónica e integral, de acuerdo con la normatividad ${ }^{(20)}$. De esta manera, los hogares sustitutos son una alternativa fundamental porque proporcionan experiencias positivas en la vida de los adolescentes con derechos vulnerados. Sin embargo, en los países desarrollados y en vías de desarrollo, si bien los centros de atención residencial subsidian las necesidades biológicas y habitacionales de los reclusos, aún no cumplen con las condiciones requeridas para el sano desarrollo de la población a la que atienden, especialmente en el ámbito emocional y psicológico ${ }^{(21)}$.

Por otro lado, para la mayoría de los adolescentes institucionalizados, sus familias no son "tan malas" y tienen la intención de volver al mismo grupo familiar ${ }^{(22)}$. Por ello, es necesario considerar la importancia de incluir a las familias en la atención brindada a estos adolescentes institucionalizados, respetando el carácter provisional y excepcional de la institución ${ }^{(23)}$. Ante ello, se recomienda establecer espacios donde sea posible conversar con la familia extensa de cada adolescente y además capacitarlos sobre los aspectos biopsicosociales del adolescente, normas de disciplina, manejo de conflictos, intervención en casos de crisis, ya que muchas veces es allí donde retornan cuando cumplen la mayoría de edad.

Por otro lado, se rescata que algunas madres rezan antes de ingerir los alimentos, enseñan los mandamientos de Dios, tienden a realizar un acercamiento a la vida espiritual, aspectos que necesitan ser investigados a mayor profundidad. También es necesario realizar estudios acerca del sentido de permanencia del vínculo, que ayuden a comprender las experiencias, sentimientos, satisfacción y expectativas que tienen los adolescentes residentes y el equipo multidisciplinario.

Una de las limitantes que se tuvo en el estudio fue la disponibilidad de tiempo de las madres cuidadoras, puesto que como tenían hijos y su trabajo era todo el día, generó que la recolección de datos durara más de los previsto. 


\section{CONCLUSIONES}

Finalmente se concluye que las madres cuidadoras de los adolescentes residentes del CAR-SJB, asumen la función como lo haría una madre biológica, coordinando con todos los profesionales que allí trabajan, y en equipo intentan formar o reformar a un adolescente separado de su familia consanguínea por orden judicial al carecer de un entorno favorable para su crecimiento y desarrollo. De este modo realizan un conjunto de actividades para satisfacer las necesidades primordiales y afectivas, inculcan disciplina y valores, y el cumplimiento del rol diario de vida para su autosuperación.

Las madres cuidadoras saben satisfacer las necesidades de alimentación e higiene, con fomento de hábitos saludables; inculcan los valores de responsabilidad, respeto, puntualidad y disciplina; brindan afecto, confianza hacia una comunicación asertiva; siguen el cumplimiento del rol de vida diario, que incluye vigilarlos sobre la realización de tareas del colegio, aprendizaje de oficios o tareas del hogar para su futura reinserción en la sociedad cuando sean mayores de edad. También propician actividades recreativas y deportivas. Incluso hay indicios que fortalecen la fe a través de la oración y actúa para coordinar siempre con todos los profesionales que allí trabajan, en especial con la enfermera, quien es la responsable de velar por la salud integral de estos adolescentes.

El aporte significativo de esta investigación permitirá dar a conocer los cuidados que se brindan considerando todas las esferas del adolescente que podrían estar afectadas, asimismo describir futuras estrategias de intervención en base a los resultados encontrados.

Se recomienda entrevistar a todo el equipo multidisciplinario, y al residente adolescente, por la necesidad de indagar cómo lograr un entorno familiar, sus expectativas, satisfacción, entre otros aspectos.

\section{AGRADECIMIENTO}

A las madres cuidadoras, por su apoyo desinteresado durante la recolección de la información.
Declaración de conflicto de intereses: Las autoras declaran no tener conflictos de interés.

\section{REFERENCIAS}

1. Ministerio de la Mujer y Poblaciones Vulnerables. Manual de Intervención en Centros de Atención Residencial de niños, niñas y adolescentes sin cuidados parentales [Internet]. 2012 [citado 18 abr 2020]; 159 p. Disponible en: https://www. mimp.gob.pe/files/direcciones/dgnna/manual_ intervencion_dgnna.pdf

2. Sánchez-Reyes JE, Cantor-Jiménez J, Castro-Sardi $\mathrm{X}$, Bolaños Y. Concepciones de niño y modos de relación de cuidadores con niños institucionalizados. Rev latinoam cienc soc niñez juv [Internet]. 2019 [citado 8 may 2020]; 17(2): 1-24. Disponible en: http://dx.doi.org/10.11600/1692715x.17209

3. Gobierno del Perú. Programa Integral Nacional para el Bienestar Familiar - INABIF - INABIF [Internet]. Familias - INABIF. 2020 [citado 8 may 2020]. Disponible en: https://www.gob.pe/inabif

4. Fernández-Daza MP, Fernández-Parra A. Problemas de comportamiento y competencias psicosociales en niños y adolescentes institucionalizados. Univ Psychol [Internet]. 2013 [citado 8 dic 2020]; 12(3). Disponible en: https://revistas.javeriana. edu.co/index.php/revPsycho/article/view/2582

5. Hueche C, Lagos G, Ríos N, Silva E, AlarcónEspinoza M. Vínculos afectivos en adolescentes institucionalizados, Chile. Rev latinoam cienc soc niñez juv [Internet]. 2019 [citado 18 dic 2020]; 17(2): 1-19. Disponible en: http://dx.doi. org/10.11600/1692715x.17217

6. Wikle J, Hoagland A. Adolescent interactions with family and emotions during interactions: Variation by family structure. J Fam Psychol [Internet]. 2020 [citado 11 abr 2020]; 34(5): 544-554. Disponible en: https://doi.org/10.1037/fam0000625

7. Vanegas J, Castrillón L. Vivencia relacional y reparación psicológica de los niños institucionalizados. Revista de Investigaciones [Internet]. 2014 [citado 11 may 2020]; 14 (23): 66-77. Disponible en: http://dx.doi.org/10.22383/ri.v14i1.8

8. Hernández-Sampieri R, Mendoza C. Metodología de la investigación: Las rutas cuantitativa, cualitativa y mixta. México: McGraw-Hill Interamericana; 2018. 714 p.

9. Mays N, Pope C, editores. Qualitative Research in Health Care. $4^{a}$ ed. Oxford: John Wiley \& Sonsdoi; 2019. 249 p.

10. Insua J. Principialismo, bioética personalista y 
principios de acción en medicina y en servicios de salud. Pers bioética [Internet]. 2018 [citado 22 jul 2020]; 22(2): 223-46. Disponible en: https://doi. org/10.5294/pebi.2018.22.2.3

11. Pérez L, Valencia L. Experiencias significativas contadas por los nińos que aportan a su proyecto de vida [Tesis de Maestría] [Internet]. Colombia: Universidad Pontificia Bolivariana; 2020 [citado 8 dic 2020]. Disponible en: https://repository.upb. edu.co/handle/20.500.11912/4967

12. Mota CP, Matos PM. Adolescents in Institutional Care: Significant Adults, Resilience and WellBeing. Child Youth Care Forum [Internet]. 2015 [citado 15 sept 2021]; 44(2): 209-224. Disponible en: https://doi.org/10.1007/s10566-014-9278-6

13. Pinchover S, Attar-Schwartz S. Institutional social climate and adjustment difficulties of adolescents in residential care: The mediating role of victimization by peers. Child Youth Serv Rev [Internet]. 2014 [citado 14 sept 2021]; 44: 393-399. Disponible en: https://doi.org/10.1016/j.childyouth.2014.07.005

14. Steels S, Simpson H, Perceptions of Children in Residential Care Homes: A Critical Review of the Literature. British Journal Social Work [Internet]. September 2017 [citado 14 sept 2021]; 47(6): 1704-1722. Disponible en: https://doi.org/10.1093/ bjsw/bcx 107

15. Mota CP, Matos PM. Caregivers' attachment and mental health: Effects on perceived bond in institutional care. Prof Psychol Res Pr [Internet]. 2016 [citado 14 sept 2021]; 47(2): 110-119. Disponible en: https://doi.org/10.1037/pro0000047

16. Martín E, González-García C, Del Valle JF, Bravo A. Detection of behavioral and emotional disorders in residential child care: Using a multi-informant approach. Child Youth Serv Rev [Internet]. 2020 [citado 13 set 2021]; 108 (104588); 1-6. Disponible en: https://doi.org/10.1016/j.childyouth. 2019.104588

17. González-García C, Bravo A, Arruabarrena I, Martín E, Santos I, Del Valle JF. Emotional and behavioral problems of children in residential care:
Screening detection and referrals to mental health services. Child Youth Serv Rev [Internet]. 2017 [citado 14 sept 2021]; 73: 100-106. Disponible en https://doi.org/10.1016/j.childyouth.2016.12.011

18. Valgañón M. Estilo de funcionamiento de las familias de acogida y conducta adaptativa - autoconcepto de los niños, niñas y adolescentes bajo su cuidado. Salud soc [Internet]. 2014 [citado 18 may 2020]; 5(2): 156-69. Disponible en: https://doi. org/10.22199/issn.0718-7475-2020-0007

19. Deambrosio M, Gutiérrez M, Arán V, Román F. Efectos del Maltrato en la Neurocognición: un estudio en niños maltratados institucionalizados y no institucionalizados. Rev latinoam cien soc niñez juv [Internet]. 2018 [citado 10 jul 2020]; 16(1): 239-53. Disponible en: http://dx.doi.org/ 10.11600/1692715x.16114

20. Vásquez V, Estrada L. Los hogares sustitutos como medida de restablecimiento de derechos de los niños, niñas y adolescentes en Colombia. Rev derecho público [Internet]. 2016 [citado 27 ago 2020]; 37(1): 1-31. Disponible en: https:// derechopublico.uniandes.edu.co/components/ com_revista/archivos/derechopub/pub586.pdf

21. Ibarra I, Romero M. Niñez y adolescencia institucionalizadas en casas hogar. Rev Elec Psic Izt [Internet]. 2017 [citado 18 ago 2020]; 20 (4): 1532-1555. Disponible en: https://www.medigra phic.com/cgi-in/new/resumen.cgi?IDARTICULO $=78430$

22. Raupp E, Accorssi, A. A prática psicopedagógica em abrigos para crianças e adolescentes. Poiésis [Internet]. 2016 [citado 21 may 2021]; 10: 216234. Disponible en: http://dx.doi.org/10.19177/ prppge.v10e02016216-234

23. Couto R, Rizzini I. Acolhimento institucional para crianças e adolescentes em situação de rua: Pesquisa e políticas públicas. Textos e Contextos [Internet]. 2021 [citado 21 may 2021]; 20(1): e39173. Disponible en: https://doi.org/10.15448/16779509.2021.1.39173 